\title{
Optimal bounds of classical and non-classical means in terms of $Q$ means
}

\section{Monika Nowicka ${ }^{1}$ (D) Alfred Witkowski ${ }^{2}$ (D)}

Received: 29 April 2021 / Accepted: 7 September 2021 / Published online: 8 October 2021

(c) The Author(s) 2021, corrected publication 2021

\section{Abstract}

We show optimal bounds of the form $Q_{\alpha}<M<Q_{\beta}$, where

$$
Q_{\alpha}(x, y)=\mathrm{A}(x, y) \frac{\mathrm{A}^{2}(x, y)}{(1-\alpha) \mathrm{A}^{2}(x, y)+\alpha \mathrm{G}^{2}(x, y)}
$$

and $M$ belongs to a broad class of classical homogeneous, symmetric means of two variables.

Keywords Classical means · Seiffert function · Q mean

Mathematics Subject Classification 26D15

\section{Introduction, definitions and notations}

aaa It is a truism to say that means are important. Means in various forms were used for practical purposes as early as in the antiquity. It is worth mentioning here the Pythagorean means of many variables (harmonic, geometric, arithmetic) or the Heronian mean. The averages have been used in practice in many areas: optics, electricity, mechanics, finance, econometrics, and statistics.

Over time, as it usually happens, the means began to live their own lives and became the subject of research of many scientists (see the list of references). In particular, the means of two variables became an interesting object of investigation. Due to big diversity of means, important information is the mutual relation between them, i.e. information about which of the three cases: $M>N, M<N$ or $M$ and $N$ are incomparable, holds. Another useful piece of information is to study the position of the mean relative to some reference family

Monika Nowicka

monika.nowicka@utp.edu.pl

Alfred Witkowski

audomat.aw@gmail.com

1 UTP University of Science and Technology Institute of Mathematics and Physics, al. prof.

Kaliskiego 7, 85-796 Bydgoszcz, Poland

2 AUDoMAT, ul. Mielczarskiego 4/29, 85-796 Bydgoszcz, Poland 
of means. The most popular reference family is the power means given by the formula $\mathrm{A}_{\mathrm{r}}(x, y)=\left(\frac{x^{r}+y^{r}}{2}\right)^{1 / r}$ and $\mathrm{A}_{0}(x, y)=\sqrt{x y}$.

Here are three examples of such results:

- for the logarithmic means Tung Po Lin [11] proved $A_{0}<L<A_{1 / 3}$,

- for the Heronian mean Alzer and Janous [1] proved optimal inequalities $A_{\log 2 / \log 3}<$ $\mathrm{He}<\mathrm{A}_{2 / 3}$,

- the result of Hästö [8] gives optimal bounds for the first Seiffert mean $A_{\log 2 / \log \pi}<P<$ $\mathrm{A}_{2 / 3}$.

In this paper we introduce a family of $Q$-means defined by

$$
Q_{\alpha}(x, y)=\mathrm{A}(x, y) \frac{\mathrm{A}^{2}(x, y)}{(1-\alpha) \mathrm{A}^{2}(x, y)+\alpha \mathrm{G}^{2}(x, y)},
$$

where $x, y>0,-4 \leq \alpha \leq \frac{1}{2}, \mathrm{~A}(x, y)=\frac{x+y}{2}$ and $\mathrm{G}(x, y)=\sqrt{x y}$ are the arithmetic and geometric means. It is easy to see that if $\alpha<\beta$ then $Q_{\alpha}<Q_{\beta}$. The $Q$-means will be used as an alternative reference line for most of the classical means as well as for some recently discovered ones. The reader will find here the optimal bounds for them in terms of $Q$-means as well as some numerical results.

Based on the shapes of the Seiffert means, one of the authors introduced in [20] the notion of Seiffert function: a function $f:(0,1) \rightarrow \mathbb{R}$ such that

$$
\mathrm{M}_{f}(x, y)= \begin{cases}\frac{|x-y|}{2 f\left(\frac{|x-y|}{x+y}\right)} & x \neq y \\ x & x=y\end{cases}
$$

is a mean. It was shown that every symmetric and homogeneous mean of positive arguments can be represented in the form (2) and that every function $f:(0,1) \rightarrow \mathbb{R}$ satisfying

$$
\frac{z}{1+z} \leq f(z) \leq \frac{z}{1-z}
$$

produces a mean. The correspondence between a mean and its Seiffert function is given by the formula

$$
f(z)=\frac{z}{M(1-z, 1+z)}, \text { where } z=\frac{|x-y|}{x+y} .
$$

In [20] it was shown also that the functions sin, tan, sinh and tanh are also Seiffert functions that produce the means called the sine, tangent, hyperbolic sine and hyperbolic tangent means.

Remark 1 Note that due to identity $\mathrm{HA}=\mathrm{G}^{2}$ (where $\mathrm{H}(x, y)=\frac{2 x y}{x+y}$ is the harmonic mean), (1) can be written as

$$
Q_{\alpha}(x, y)=\mathrm{A}(x, y) \frac{\mathrm{A}(x, y)}{(1-\alpha) \mathrm{A}(x, y)+\alpha \mathrm{H}(x, y)} .
$$

Let us use the formula (4) to calculate the Seiffert function of $Q_{\alpha}$ :

$$
\begin{aligned}
q_{\alpha}(z) & =\frac{z}{\mathrm{~A}(1+z, 1-z) \frac{\mathrm{A}(1+z, 1-z)}{(1-\alpha) \mathrm{A}(1+z, 1-z)+\alpha \mathrm{H}(1+z, 1-z)}} \\
& =z\left(1-\alpha+\alpha\left(1-z^{2}\right)\right)=z-\alpha z^{3} .
\end{aligned}
$$


In this paper we shall establish the optimal bounds of the form $Q_{\alpha}<M<Q_{\beta}$, where $M$ is one of the classical (or less classical) means.

Remark 2 For two means $M, N$ the notation $M<N$ means that $M(x, y)<N(x, y)$ holds unless $x=y$.

Remark 3 If $m, n$ are the Seiffert functions of $M, N$ respectively, then the inequality $M<N$ is equivalent to $n<m$ by the formula (2).

Note that the inequalities $Q_{\alpha}<\mathrm{M}<Q_{\beta}$ in terms of Seiffert functions can be rewritten as $q_{\beta}(z)<m(z)<q_{\alpha}(z)$, and, given (5), to find optimal $\alpha$ and $\beta$ we may use one of the three methods

Method 1 Evaluate

$$
\begin{aligned}
& \alpha=\inf _{0<z<1} \frac{z-m(z)}{z^{3}}, \\
& \beta=\sup _{0<z<1} \frac{z-m(z)}{z^{3}},
\end{aligned}
$$

or

Method 2 Calculate

$$
\begin{aligned}
& \alpha=\sup \left\{\gamma: z-\gamma z^{3}-m(z) \geq 0 \text { holds for all } z \in(0,1)\right\}, \\
& \beta=\inf \left\{\gamma: z-\gamma z^{3}-m(z) \leq 0 \text { holds for all } z \in(0,1)\right\},
\end{aligned}
$$

or equivalently

Method 3 Calculate

$$
\begin{aligned}
& \alpha=\sup \left\{\gamma: 1-\gamma z^{2}-\frac{m(z)}{z} \geq 0 \text { holds for all } z \in(0,1)\right\}, \\
& \beta=\inf \left\{\gamma: 1-\gamma z^{2}-\frac{m(z)}{z} \leq 0 \text { holds for all } z \in(0,1)\right\} .
\end{aligned}
$$

\section{Remarks on $Q$ means}

It might be surprising why we consider the $Q_{\alpha}$ means for $-4 \leq \alpha \leq \frac{1}{2}$ only. The following lemmas give the answer.

Lemma 1 The function $q_{\alpha}(z)=z-\alpha z^{3}$ is a Seiffert function if and only if $-4 \leq \alpha \leq \frac{1}{2}$.

Proof The inequalities (3) can be rewritten as

$$
-\frac{1}{z(1-z)} \leq \alpha \leq \frac{1}{z(1+z)}
$$

and since $\inf _{z \in(0,1)} \frac{1}{z(1+z)}=\frac{1}{2}$ and $\max _{z \in(0,1)} \frac{-1}{z(1-z)}=-4$, the assertion holds.

Lemma 2 The function $q_{\alpha}(z)=z-\alpha z^{3}$ is the Seiffert function of $Q_{\alpha}$.

Proof By (4) we have

$$
\frac{z}{Q_{\alpha}(1+z, 1-z)}=\frac{z}{\frac{1}{1-\alpha+\alpha\left(1-z^{2}\right)}}=\mathrm{q}_{\alpha}(z) .
$$




\section{Main results}

At the beginning we consider means that are lesser than the logarithmic mean:

- harmonic mean $\mathrm{H}(x, y)=\frac{2 x y}{x+y}=\mathrm{A}_{-1}(x, y)$. Its Seiffert function equals $\mathrm{h}(z)=\frac{z}{1-z^{2}}$,

- power mean of order $-1 / 2, \mathrm{~A}_{-1 / 2}(x, y)=\left(\frac{x^{-1 / 2}+y^{-1 / 2}}{2}\right)^{-2}$ with Seiffert function $\mathrm{a}_{-1 / 2}(z)=\frac{z\left(1+\sqrt{1-z^{2}}\right)}{2\left(1-z^{2}\right)}$,

- geometric mean $\mathrm{G}(x, y)=\sqrt{x y}$ with $\mathrm{g}(z)=\frac{z}{\sqrt{1-z^{2}}}$,

- logarithmic mean $\mathrm{L}(x, y)=\frac{x-y}{\ln x-\ln y}=\mathrm{M}_{\operatorname{artanh}}(x, y)=\frac{|x-y|}{2 \operatorname{artanh} \frac{|x-y|}{x+y}}$. The last formula shows that $\mathrm{I}(z)=\operatorname{artanh} z$.

All the means mentioned above have one common property: $\lim _{x \rightarrow 0^{+}} M(x, 1)=0$, which indicates that there is no lower bound for them in the class of $Q$ means, as the limit of $Q_{\alpha}$ at zero is positive.

Theorem 1 (Bounds for harmonic mean) The inequality

$$
H(x, y) \leq Q_{\beta}(x, y)
$$

holds if and only if $\beta \geq-1$.

Proof Consider the function from Method 1:

$$
u(z)=\frac{z-\frac{z}{1-z^{2}}}{z^{3}}=-\frac{1}{1-z^{2}} .
$$

It decreases and assumes values in $(-\infty,-1)$, which completes the proof.

Theorem 2 (Bounds for power mean of order $-\frac{1}{2}$ ) The inequality

$$
A_{-1 / 2}(x, y) \leq Q_{\beta}(x, y)
$$

holds if and only if $\beta \geq-\frac{3}{4}$. There is no lower bound for the power mean of order $-1 / 2$ in the family of $Q$ means.

Proof Consider the function in Method 3

$$
g_{\gamma}(z)=1-\gamma z^{2}-\frac{1}{2}\left(\frac{1}{1-z^{2}}+\frac{1}{\sqrt{1-z^{2}}}\right) .
$$

The function $g_{\gamma}$ satisfies $g_{\gamma}(0)=0$ and (as every even function) $g_{\gamma}^{\prime}(0)=0$. Moreover

$$
g_{\gamma}^{\prime \prime}(z)=-2 \gamma-\frac{1}{2}\left(\frac{6 z^{2}+2}{\left(1-z^{2}\right)^{3}}+\frac{2 z^{2}+1}{\left(1-z^{2}\right)^{5 / 2}}\right) .
$$

Both fractions in (6) increase (numerators increase and denominators decrease) thus $g_{\gamma}^{\prime \prime}$ decreases from $-2 \gamma-\frac{3}{2}$ to $-\infty$. This implies that if $\gamma \geq-\frac{3}{4}$ the function $g_{\gamma}$ is concave and therefore satisfies $g_{\gamma}(z)<0$ for $0<z<1$. If $\gamma<-\frac{3}{4}$ the function $g_{\gamma}$ is convex and thus positive for small $z$, and cannot preserve sign, because it tends to $-\infty$ at the right end. $\square$

Theorem 3 (Bounds for geometric mean) The inequality $G<Q_{\beta}$ holds if and only if $\beta \geq$ $-1 / 2$. There is no $Q_{\alpha}$ that bounds the geometric mean from below. 
Proof We use Method 1. The function

$$
\frac{z-\frac{z}{\sqrt{1-z^{2}}}}{z^{3}}=\frac{\sqrt{1-z^{2}}-1}{z^{2} \sqrt{1-z^{2}}}=-\frac{1}{\sqrt{1-z^{2}}\left(1+\sqrt{1-z^{2}}\right)}
$$

decreases because both functions in denominator decrease. Therefore it assumes values in $\left(-\infty,-\frac{1}{2}\right)$, which completes the proof.

Let us recall that the Seiffert function of the logarithmic mean $\mathrm{I}(z)=\operatorname{artanh} z$.

Theorem 4 (Bounds for logarithmic mean). The inequalities $M_{\operatorname{artanh}}<Q_{\beta}$ hold if and only if $\beta \geq-\frac{1}{3} \approx-0.3333$. There is no $Q_{\alpha}$ that bounds the logarithmic mean from below.

Proof Let us consider the function from Method 2: $u_{\gamma}(z)=z-\gamma z^{3}-\operatorname{artanh} z$. It is elementary that $u_{\gamma}(0)=u_{\gamma}^{\prime}(0)=0$. Let us investigate the sign of its second derivative.

$$
u_{\gamma}^{\prime \prime}(z)=-6 \gamma z-\frac{2 z}{\left(1-z^{2}\right)^{2}}=-2 z\left(3 \gamma+\frac{1}{\left(1-z^{2}\right)^{2}}\right) .
$$

It is easy to see that if $\gamma \geq-\frac{1}{3}$, then $u_{\gamma}$ is concave and thus negative for all $z \in(0,1)$. For $\gamma<-\frac{1}{3}$ our function is convex and positive for small $z$ and negative for $z$ close to 1 , which means that it cannot preserve sign, thus $\alpha$ is not a real number.

There are many interesting means between the logarithmic and the arithmetic means. We shall consider five of them. Here they are:

- power mean of order $\frac{1}{2}, \mathrm{~A}_{1 / 2}(x, y)=\left(\frac{\sqrt{x}+\sqrt{y}}{2}\right)^{2}$ with Seiffert function $\mathrm{a}_{1 / 2}(z)=$ $\frac{2 z}{1+\sqrt{1-z^{2}}}$,

- Heronian mean $\mathrm{He}(x, y)=\frac{x+\sqrt{x y}+y}{3}$ used to calculate the volume of a truncated pyramid long before Christ. Its Seiffert function equals he $(z)=\frac{3 z}{2+\sqrt{1-z^{2}}}$,

- tangent mean $\mathrm{M}_{\tan }(x, y)=\frac{|x-y|}{2 \tan \frac{|x-y|}{x+y}}$. Obviously, tangent is its Seiffert function,

- arcsine or first Seiffert mean $\mathrm{P}(x, y)=\mathrm{M}_{\arcsin }(x, y)=\frac{|x-y|}{2 \arcsin \frac{|x-y|}{x+y}}$ with $\mathrm{p}(z)=\arcsin z$,

- hyperbolic sine mean $\mathrm{M}_{\sinh }(x, y)=\frac{|x-y|}{2 \sinh \frac{|x-y|}{x+y}}$ with $\mathrm{m}_{\sinh }(z)=\sinh z$.

The following inequalities between these means can be found in the literature.

Inequality $L<A_{1 / 3}$ has been proven in [11]. This combined with monotonicity of power means shows that $L<A_{1 / 2}$.

Inequalities

$$
L<\left\{\begin{array}{c}
\mathrm{P} \\
\mathrm{M}_{\tan }
\end{array}\right\}<\mathrm{M}_{\text {sinh }}<\mathrm{A}
$$

have been proven in [20] (the means in curly brackets are not comparable). The means $A_{1 / 2}$ and $M_{\tan }$ are not comparable either, and the relations

$$
\mathrm{A}_{1 / 2}<\mathrm{P}<\mathrm{He}
$$

has been proved by Hastö in [8] (left part) and Sándor in [15] (right part).

Finally

$$
\mathrm{M}_{\mathrm{tan}}<\mathrm{He}<\mathrm{M}_{\text {sinh }}
$$


follow from Theorems 2.1 and 2.2 in [12]. Summarizing, we have the following chain of inequalities:

$$
L<\left\{\begin{array}{c}
A_{1 / 2}<P \\
M_{\tan }
\end{array}\right\}<H e<M_{\text {sinh }}<A .
$$

Let us begin with the Heronian mean $\mathrm{He}=\frac{2 \mathrm{~A}+\mathrm{G}}{3}$ and the power mean of order $\frac{1}{2}$ that can be written as $A_{1 / 2}=\frac{A+G}{2}$. Both are members of the family of means that interpolate between the geometric and the arithmetic means given by $\mathrm{He}_{p}=(1-p) \mathrm{G}+p \mathrm{~A}, 0 \leq p \leq 1$. Obviously $\mathrm{He}=\mathrm{He}_{2 / 3}$. The Seiffert function for $\mathrm{He}_{p}$ is

$$
\text { he }_{p}(z)=\frac{z}{(1-p) \sqrt{1-z^{2}}+p} \text {. }
$$

Theorem 5 (Bounds for the Heronian family of means). Let $0 \leq p \leq 1$. The inequalities $\mathrm{He}_{p}<Q_{\beta}$ hold if and only if $\beta \geq \frac{p-1}{2}$.

If $p \geq \frac{1}{5}$, then the inequality $Q_{\alpha}<H e_{p}$ holds if and only if $\alpha \leq \frac{p-1}{p}$.

If $p<\frac{1}{5}$, then there is no lower bound for $\mathrm{He}_{p}$.

Proof We shall use Method 1 here:

$$
\frac{z-\text { he }_{p}(z)}{z^{3}}=\frac{p-1}{\left[(1-p) \sqrt{1-z^{2}}+p\right]\left[\sqrt{1-z^{2}}+1\right]},
$$

which shows that this function decreases with $z \in(0,1)$ and assumes values in $\left(\frac{p-1}{p}, \frac{p-1}{2}\right)$. For $p<\frac{1}{5}$, we have $(p-1) / p<-4$, so the corresponding $Q$ is not a mean by Lemma 1 .

Corollary 1 The optimal inequalities hold:

$$
Q_{-1}<A_{1 / 2}<Q_{-1 / 4}
$$

Corollary 2 The optimal inequalities hold:

$$
Q_{-1 / 2}<\mathrm{He}<Q_{-1 / 6} .
$$

And now it's time for three other means

Theorem 6 (Bounds for tangent mean) The inequalities $Q_{\alpha}<M_{\tan }<Q_{\beta}$ hold if and only if $\alpha \leq 1-\tan 1 \approx-0.5574$ and $\beta \geq-\frac{1}{3} \approx-0.3333$.

Proof Consider the function from Method 2:

$$
g_{\gamma}(z)=z-\gamma z^{3}-\tan z
$$

It satisfies $g_{\gamma}(0)=g_{\gamma}^{\prime}(0)=0$ and $g_{\gamma}(1)=1-\gamma-\tan 1$. Moreover,

$$
g_{\gamma}^{\prime \prime}(z)=-2 z\left(3 \gamma+\frac{\sin z}{z \cos ^{3} z}\right) .
$$

The function $\frac{\sin z}{\cos ^{3} z}=\frac{1}{\cos z} \cdot \frac{1}{\cos z} \cdot \tan z$ is a product of three positive, increasing and convex functions, so it is convex. By Property 2 its divided difference $\frac{\sin z}{z \cos ^{3} z}$ increases from $m=1$ to $M=\frac{\sin 1}{\cos ^{3} 1}$. Thus we see that if $3 \gamma+1 \geq 0$, then $g_{\gamma}$ is concave and therefore negative. On the other hand, if $3 \gamma+1<0$ then $g_{\gamma}$ is convex, and thus positive for small values of $z$ and changes its convexity at most once. Therefore it remains nonnegative in the unit interval if and only if $g_{\gamma}(1)=1-\gamma-\tan 1 \geq 0$. 
Theorem 7 (Bounds for first Seiffert mean). The inequalities $Q_{\alpha}<P<Q_{\beta}$ hold if and only if $\alpha \leq 1-\pi / 2 \approx-0.5708$ and $\beta \geq-\frac{1}{6} \approx-0.1667$.

Proof We shall use once more Method 2 and investigate

$$
g_{\gamma}(z)=z-\gamma z^{3}-\arcsin z
$$

It satisfies $g_{\gamma}(0)=g_{\gamma}^{\prime}(0)=0$ and $g_{\gamma}(1)=1-\gamma-\arcsin 1$. Moreover,

$$
g_{\gamma}^{\prime \prime}(z)=-z\left(6 \gamma+\frac{1}{\left(1-z^{2}\right)^{3 / 2}}\right) \text {. }
$$

The function $6 \gamma+\frac{1}{\left(1-z^{2}\right)^{3 / 2}}$ increases from $6 \gamma+1$ to infinity, so if $6 \gamma+1 \geq 0$ the function $g_{\gamma}$ is concave, and thus negative, while if $6 \gamma+1<0$, the function $g_{\gamma}$ is convex and positive for small $z$ and then becomes concave. As a consequence it remains positive on $(0,1)$ if and only if $g_{\gamma}(1) \geq 0$.

Theorem 8 (Bounds for hyperbolic sine mean). The inequalities $Q_{\alpha}<M_{\sinh }<Q_{\beta}$ hold if and only if $\alpha \leq 1-\sinh 1 \approx-0.1752$ and $\beta \geq-\frac{1}{6} \approx-0.1667$.

Proof We use again Method 2 and investigate:

$$
g_{\gamma}(z)=z-\gamma z^{3}-\sinh z .
$$

It satisfies $g_{\gamma}(0)=g_{\gamma}^{\prime}(0)=0$ and $g_{\gamma}(1)=1-\gamma-\sinh 1$. Moreover,

$$
g_{\gamma}^{\prime \prime}(z)=-z\left(6 \gamma+\frac{\sinh z}{z}\right) .
$$

The function $\frac{\sinh z}{z}$ increases by Property 2 from 1 to infinity, so if $6 \gamma+1 \geq 0$ the function $g_{\gamma}$ is concave, and thus negative, while if $6 \gamma+1<0$ the function $g_{\gamma}$ is convex and positive for small $z$ and then remains convex or becomes concave. As a consequence it remains positive on $(0,1)$ if and only if $g_{\gamma}(1) \geq 0$.

Next we shall take care of several means that are greater than the arithmetic mean:

- Neuman-Sándor mean called also inverse hyperbolic sine mean NS $(x, y)=\frac{|x-y|}{2 \operatorname{arsinh} \frac{|x-y|}{x+y}}$ with Seiffert function $\mathrm{ns}(z)=\operatorname{arsinh} z$,

- sine mean $\mathrm{M}_{\sin }(x, y)=\frac{|x-y|}{2 \sin \frac{|x-y|}{x+y}}$,

- second Seiffert mean or arctangent mean $\mathrm{T}(x, y)=\mathrm{M}_{\arctan }(x, y)=\frac{|x-y|}{2 \arctan \frac{|x-y|}{x+y}}$,

- hyperbolic tangent mean $\mathrm{M}_{\tanh }(x, y)=\frac{|x-y|}{2 \tanh \frac{|x-y|}{x+y}}$,

- centroidal mean $\operatorname{Ce}(x, y)=\frac{2}{3} \frac{x^{2}+x y+y^{2}}{x+y}$. Its $\operatorname{Seffert}$ mean is $\operatorname{ce}(z)=\frac{3 z}{3+z^{2}}$,

- root-mean square or quadratic mean $\operatorname{RMS}(x, y)=\sqrt{\frac{x^{2}+y^{2}}{2}}$ with Seiffert function $\operatorname{rms}(z)=\frac{z}{\sqrt{1+z^{2}}}$,

- contraharmonic mean $\mathrm{C}(x, y)=\frac{x^{2}+y^{2}}{x+y}$ where $\mathrm{C}(z)=\frac{z}{1+z^{2}}$.

We have

$$
\mathrm{A}<\mathrm{NS}<\mathrm{M}_{\sin }<\mathrm{T}<\mathrm{M}_{\tanh }<\mathrm{Ce}<\mathrm{RMS}<\mathrm{C} .
$$

The first four inequalities come from [20], next from [13], the remaining two can be found in many sources.

In this part of our paper Method 1 proves to be very useful. 
Theorem 9 (Bounds for Neuman-Sándor mean). The inequalities $Q_{\alpha}<M_{\operatorname{arsinh}}<Q_{\beta}$ hold if and only if $\alpha \leq 1-\operatorname{arsinh} 1 \approx 0.1186$ and $\beta \geq \frac{1}{6} \approx 0.1667$.

Proof The function

$$
\frac{(z-\operatorname{arsinh} z)^{\prime}}{\left(z^{3}\right)^{\prime}}=\frac{1-\frac{1}{\sqrt{1+z^{2}}}}{3 z^{2}}=\frac{1}{3 \sqrt{1+z^{2}}\left(1+\sqrt{1+z^{2}}\right)}
$$

decreases, so by Lemma $3 h(z)=\frac{z-\operatorname{arsinh} z}{z^{3}}$ decreases, assuming values between $\lim _{z \rightarrow 0^{+}} h(z)=\frac{1}{6}$ and $h(1)=1-\operatorname{arsinh} 1$, which completes the proof.

Theorem 10 (Bounds for the sine mean). The inequalities $Q_{\alpha}<M_{\sin }<Q_{\beta}$ hold if and only if $\alpha \leq 1-\sin 1 \approx 0.1585$ and $\beta \geq \frac{1}{6} \approx 0.1667$.

Proof Applying Lemma 3 to the functions $f(z)=z-\sin z$ and $g(z)=z^{3}$ we see that

$$
\frac{f^{\prime}(z)}{g^{\prime}(z)}=\frac{1-\cos z}{3 z^{2}}=\frac{\sin ^{2}(z / 2)}{6(z / 2)^{2}}
$$

decreases thus $f(z) / g(z)$ does so. Thus $h(z)=\frac{z-\sin z}{z^{3}}$ from Method 1 assumes values between $\lim _{z \rightarrow 0^{+}} h(z)=\frac{1}{6}$ and $h(1)=1-\sin 1$.

Theorem 11 (Bounds for the second Seiffert mean). The inequalities $Q_{\alpha}<M_{\arctan }<Q_{\beta}$ hold if and only if $\alpha \leq 1-\frac{\pi}{4} \approx 0.2146$ and $\beta \geq \frac{1}{3} \approx 0.3333$.

Proof Applying Lemma 3 to the functions $f(z)=z-\arctan z$ and $g(z)=z^{3}$ we see that

$$
\frac{f^{\prime}(z)}{g^{\prime}(z)}=\frac{1-\frac{1}{1+z^{2}}}{3 z^{2}}=\frac{1}{3} \frac{1}{1+z^{2}}
$$

decreases and $f(z) / g(z)$ does so. Consequently, $h(z)=\frac{z-\arctan z}{z^{3}}$ assumes values between $\lim _{z \rightarrow 0^{+}} h(z)=\frac{1}{3}$ and $h(1)=1-\frac{\pi}{4}$.

Theorem 12 (Bounds for the hyperbolic tangent mean). The inequalities $Q_{\alpha}<M_{\tanh }<Q_{\beta}$ hold if and only if $\alpha \leq 1-\tanh 1 \approx 0.2384$ and $\beta \geq \frac{1}{3} \approx 0.3333$.

Proof To show that $h(z)=\frac{z-\tanh z}{z^{3}}$ decreases we use Lemma 3. We have

$$
\frac{(z-\tanh z)^{\prime}}{\left(z^{3}\right)^{\prime}}=\frac{1}{3}\left(\frac{\tanh z}{z}\right)^{2} .
$$

The function tanh is concave and $\lim _{z \rightarrow 0^{+}} \tanh z=0$, so by Property 2 the function $\frac{\tanh z}{z}$ decreases and this implies that the function $h$ used in Method 1 decreases. We complete the proof by noting $\lim _{z \rightarrow 0^{+}} h(z)=\frac{1}{3}$.

Theorem 13 (Bounds for centroidal mean). The inequalities $Q_{\alpha}<\mathrm{Ce}<Q_{\beta}$ hold if and only if $\alpha \leq \frac{1}{4}$ and $\beta \geq \frac{1}{3}$.

Proof The function

$$
h(z)=\frac{z-\frac{3 z}{3+z^{2}}}{z^{3}}=\frac{1}{3+z^{2}}
$$

decreases, so it assumes values between $h(1)=\frac{1}{4}$ and $h(0)=\frac{1}{3}$. 
Table 1 Absolute errors

\begin{tabular}{|c|c|c|c|c|c|}
\hline \multirow[b]{2}{*}{ Mean } & \multirow[b]{2}{*}{$\alpha$} & \multicolumn{2}{|c|}{$\sup M-Q_{\alpha}$} & \multicolumn{2}{|c|}{$\sup Q_{\beta}-M$} \\
\hline & & value & at $x=$ & $\beta$ & value at $0^{+}$ \\
\hline Harmonic mean & & $\mathrm{n} / \mathrm{a}$ & & -1 & $\frac{1}{4}=0.25$ \\
\hline Power mean of order $-1 / 2$ & & $\mathrm{n} / \mathrm{a}$ & & $-\frac{3}{4}$ & $\frac{2}{7} \approx 0.2857$ \\
\hline Geometric mean & & $\mathrm{n} / \mathrm{a}$ & & $-\frac{1}{2}$ & $\frac{1}{3} \approx 0.3333$ \\
\hline Logarithmic mean & & $\mathrm{n} / \mathrm{a}$ & & $-\frac{1}{3}$ & $\frac{3}{8}=0.375$ \\
\hline Power mean of order $1 / 2$ & -1 & 0.1095 & 0.1642 & $-\frac{1}{4}$ & $\frac{3}{20}=0.15$ \\
\hline Heronian mean & $-\frac{1}{2}$ & 0.0610 & 0.1327 & $-\frac{1}{6}$ & $\frac{2}{21} \approx 0.0952$ \\
\hline Tangent mean & $1-\tan 1$ & 0.0277 & 0.2275 & $-\frac{1}{3}$ & $\frac{3}{8}-\frac{1}{2 \tan 1} \approx 0.0540$ \\
\hline First Seiffert mean & $1-\frac{\pi}{2}$ & 0.0729 & 0.1330 & $-\frac{1}{6}$ & $\frac{3}{7}-\frac{1}{\pi} \approx 0.1103$ \\
\hline Hyperbolic sine mean & $1-\sinh 1$ & 0,0011 & 0.2218 & $-\frac{1}{6}$ & $\frac{3}{7}-\frac{1}{2 \sinh 1} \approx 0.0031$ \\
\hline Neuman-Sándor mean & $1-\operatorname{arsinh} 1$ & 0.0065 & 0.2126 & $\frac{1}{6}$ & $\frac{3}{5}-\frac{1}{2 \operatorname{arsinh} 1} \approx 0.0327$ \\
\hline Sine mean & $1-\sin 1$ & 0.0014 & 0.1778 & $\frac{1}{6}$ & $\frac{3}{5}-\frac{1}{2 \sin 1} \approx 0.0058$ \\
\hline Second Seiffert mean & $1-\arctan 1$ & 0.0170 & 0.2017 & $\frac{1}{3}$ & $\frac{3}{4}-\frac{1}{2 \arctan 1} \approx 0.1134$ \\
\hline Hyperbolic tangent mean & $1-\tanh 1$ & 0.0153 & 0.1859 & $\frac{1}{3}$ & $\frac{3}{4}-\frac{1}{2 \tanh 1} \approx 0.0935$ \\
\hline Centroidal mean & $\frac{1}{4}$ & 0.0140 & 0.1802 & $\frac{1}{3}$ & $\frac{1}{12} \approx 0.8333$ \\
\hline Quadratic mean & $\frac{1}{2+\sqrt{2}}$ & 0.0313 & 0.1924 & $\frac{1}{2}$ & $\frac{\sqrt{2}-1}{\sqrt{2}} \approx 0.2929$ \\
\hline
\end{tabular}

All the maxima in the rightmost column occur at $0^{+}$

Theorem 14 (Bounds for quadratic mean). The inequalities $Q_{\alpha}<R M S<Q_{\beta}$ hold if and only if $\alpha \leq \frac{1}{2+\sqrt{2}} \approx 0.2929$ and $\beta \geq \frac{1}{2}$.

Proof Once more we use Method 1. The function

$$
h(z)=\frac{z-\frac{z}{\sqrt{1+z^{2}}}}{z^{3}}=\frac{\sqrt{1+z^{2}}-1}{z^{2} \sqrt{1+z^{2}}}=\frac{1}{\sqrt{1+z^{2}}\left(1+\sqrt{1+z^{2}}\right)}
$$

decreases, so it assumes values between $h(1)=\frac{1}{2+\sqrt{2}}$ and $h(0)=\frac{1}{2}$.

\section{Error rates}

Tables 1 on page 9 and Table 2 on page 10 represent absolute and relative errors of the bounds calculated in the preceding section.

\section{Remarks about other one-parameter families of means}

In this section we consider two more families of one-parameter means given by formulae similar to (1) and $\left(1^{\prime}\right)$

$$
V_{\alpha}=\mathrm{A} \frac{\mathrm{A}^{2}}{(1-\alpha) \mathrm{A}^{2}+\alpha \mathrm{RMS}^{2}}=\mathrm{A} \frac{\mathrm{A}}{(1-\alpha) \mathrm{A}+\alpha \mathrm{C}},
$$


Table 2 Relative errors (in percents)

\begin{tabular}{|c|c|c|c|c|c|}
\hline \multirow[b]{2}{*}{ Mean } & \multirow[b]{2}{*}{$\alpha$} & \multicolumn{2}{|l|}{$\sup \frac{M-Q_{\alpha}}{M}$} & \multicolumn{2}{|c|}{$\sup \frac{Q_{\beta}-M}{M}$} \\
\hline & & value $[\%]$ & at $x=$ & $\beta$ & value $[\%]$ at $0^{+}$ \\
\hline Power mean of order $1 / 2$ & -1 & 23.99 & 0.0893 & $-\frac{1}{4}$ & 60.00 \\
\hline Heronian mean & $-\frac{1}{2}$ & 12.78 & 0.0842 & $-\frac{1}{6}$ & 28.57 \\
\hline Tangent mean & $1-\tan 1$ & 5.50 & 0.1670 & $-\frac{1}{3}$ & 16.81 \\
\hline First Seiffert mean & $1-\frac{\pi}{2}$ & 15.37 & 0.0820 & $-\frac{1}{6}$ & 34.64 \\
\hline Hyperbolic sine mean & $1-\sinh 1$ & 0.20 & 0.1801 & $-\frac{1}{6}$ & 0.73 \\
\hline Neuman-Sándor mean & $1-\operatorname{arsinh} 1$ & 1.01 & 0.1899 & $\frac{1}{6}$ & 5.76 \\
\hline Sine mean & $1-\sin 1$ & 0.22 & 0.1627 & $\frac{1}{6}$ & 0.98 \\
\hline Second Seiffert mean & $1-\arctan 1$ & 2.51 & 0.1876 & $\frac{1}{3}$ & 17.81 \\
\hline Hyperbolic tangent mean & $1-\tanh 1$ & 2.24 & 0.1757 & $\frac{1}{3}$ & 14.24 \\
\hline Centroidal mean & $\frac{1}{4}$ & 2.04 & 0.1716 & $\frac{1}{3}$ & 12.50 \\
\hline Quadratic mean & $\frac{1}{2+\sqrt{2}}$ & 4.35 & 0.1858 & $\frac{1}{2}$ & 41.42 \\
\hline
\end{tabular}

All the maxima in the rightmost column occur at $0^{+}$

where $\operatorname{RMS}(x, y)=\sqrt{\frac{x^{2}+y^{2}}{2}}$ is the quadratic (or root-mean square) mean and $\mathrm{C}(x, y)=$ $\frac{x^{2}+y^{2}}{x+y}$ is the contraharmonic mean. Using (4) we see that the corresponding Seiffert functions are of the form $z+\alpha z^{3}$, so by Lemma $1 V_{\alpha}$ are means if and only if $-\frac{1}{2} \leq \alpha \leq 4$ and $V_{\alpha}=Q_{-\alpha}$.

The second family is

$$
W_{\alpha}=\mathrm{A} \frac{\mathrm{A}}{(1-\alpha) \mathrm{A}+\alpha \mathrm{Ce}},
$$

where $\mathrm{Ce}(x, y)=\frac{2}{3} \frac{x^{2}+x y+y^{2}}{x+y}$ is the centroidal mean. We use (4) to find out that their Seiffert functions are of the form $w_{\alpha}(z)=z+\frac{\alpha}{3} z^{3}$. Again by Lemma 1 we see that $W_{\alpha}$ are means if and only if $-\frac{3}{2} \leq \alpha \leq 12$ and $W_{\alpha}=Q_{\alpha / 3}$.

\section{Tools and lemmas}

In this section, we place all the technical details needed to prove our main results.

Property 1 A function $f:(a, b) \rightarrow \mathbb{R}$ is convex if, and only if, for every $a<\theta<b$ its divided difference $\frac{f(x)-f(\theta)}{x-\theta}$ increases for $x \neq \theta$.

A simple consequence of Property 1 is

Property 2 If a function $f:(a, b) \rightarrow \mathbb{R}$ is convex and $\lim _{x \rightarrow a} f(x)=\Theta$, then the function $\frac{f(x)-\Theta}{x-a}$ increases.

The next lemma can be found in [2, Theorem 1.25].

Lemma 3 Suppose $f, g:(a, b) \rightarrow \mathbb{R}$ are differentiable with $g^{\prime}(x) \neq 0$ and such that $\lim _{x \rightarrow a} f(x)=\lim _{x \rightarrow a} g(x)=0$ or $\lim _{x \rightarrow b} f(x)=\lim _{x \rightarrow b} g(x)=0$. Then 
1. if $\frac{f^{\prime}}{g^{\prime}}$ is increasing on $(a, b)$, then $\frac{f}{g}$ is increasing on $(a, b)$,

2. if $\frac{f^{\prime}}{g^{\prime}}$ is decreasing on $(a, b)$, then $\frac{f}{g}$ is decreasing on $(a, b)$.

Acknowledgements The authors wish to thank the anonymous referee for their careful reading of the manuscript. We are grateful for their suggestions to add references [3-7,9,10,14,16-19], that present the main research content of the article, and the research background and research progress in this field.

\section{Declarations}

Conflict of interest The authors declare that they have no conflict of interest.

Open Access This article is licensed under a Creative Commons Attribution 4.0 International License, which permits use, sharing, adaptation, distribution and reproduction in any medium or format, as long as you give appropriate credit to the original author(s) and the source, provide a link to the Creative Commons licence, and indicate if changes were made. The images or other third party material in this article are included in the article's Creative Commons licence, unless indicated otherwise in a credit line to the material. If material is not included in the article's Creative Commons licence and your intended use is not permitted by statutory regulation or exceeds the permitted use, you will need to obtain permission directly from the copyright holder. To view a copy of this licence, visit http://creativecommons.org/licenses/by/4.0/.

\section{References}

1. Alzer, H., Janous, W.: Solution of problem 8*. Crux. Math. 13, 173-178 (1987)

2. Anderson, G.D., Vamanamurthy, M.K., Vuorinen, M.K.: Conformal Invariants, Inequalities, and Quasiconformal Maps. Canadian Mathematical Society Series of Monographs and Advanced Texts. Wiley, New York (1997)

3. Chu, H.H., Zhao, T.H., Chu, Y.M.: Sharp bounds for the Toader mean of order 3 in terms of arithmetic, quadratic and contraharmonic means. Math. Slovaca 70(5), 1097-1112 (2020). https://doi.org/10.1515/ ms-2017-0417

4. Chu, Y.M., Qiu, Y.F., Wang, M.K.: Hölder mean inequalities for the complete elliptic integrals. Integral Transforms Spec. Funct. 23(7), 521-527 (2012). https://doi.org/10.1080/10652469.2011.609482

5. Chu, Y.M., Wang, M.K.: Optimal Lehmer mean bounds for the Toader mean. Results Math. 61(3-4), 223-229 (2012). https://doi.org/10.1007/s00025-010-0090-9

6. Chu, Y.M., Wang, M.K., Qiu, S.L.: Optimal combinations bounds of root-square and arithmetic means for Toader mean. Proc. Indian Acad. Sci. Math. Sci. 122(1), 41-51 (2012). https://doi.org/10.1007/s12044012-0062-y

7. Chu, Y.M., Wang, M.K., Wang, Z.K.: Best possible inequalities among harmonic, geometric, logarithmic and Seiffert means. Math. Inequal. Appl. 15(2), 415-422 (2012). https://doi.org/10.7153/mia-15-36

8. Hästö, P.A.: Optimal inequalities between Seiffert's mean and power means. Math. Inequal. Appl. 7(1), 47-53 (2004). https://doi.org/10.7153/mia-07-06

9. He, X.H., Qian, W.M., Xu, H.Z., Chu, Y.M.: Sharp power mean bounds for two Sándor-Yang means. Rev. R. Acad. Cienc. Exactas Fís. Nat. Ser. A Mat. RACSAM 113(3), 2627-2638 (2019). https://doi.org/ 10.1007/s13398-019-00643-2

10. He, Z.Y., Wang, M.K., Jiang, Y.P., Chu, Y.M.: Bounds for the perimeter of an ellipse in terms of power means. J. Math. Inequal. 14(3), 887-899 (2020). https://doi.org/10.7153/jmi-2020-14-58

11. Lin, T.P.: The power mean and the logarithmic mean. Am. Math. Mon. 81, 879-883 (1974). https://doi. org/10.2307/2319447

12. Nowicka, M., Witkowski, A.: Optimal bounds for the tangent and hyperbolic sine means. Aequ. Math. 94(5), 817-827 (2020). https://doi.org/10.1007/s00010-020-00705-6

13. Nowicka, M., Witkowski, A.: Optimal bounds for the sine and hyperbolic tangent means IV. Rev. R. Acad. Cienc. Exactas Fís. Nat. Ser. A Mat. RACSAM 115(2), 79 (2021). https://doi.org/10.1007/s13398-02101020-8

14. Qian, W.M., Zhang, W., Chu, Y.M.: Bounding the convex combination of arithmetic and integral means in terms of one-parameter harmonic and geometric means. Miskolc Math. Notes 20(2), 1157-1166 (2019)

15. Sándor, J.: On certain inequalities for means III. Arch. Math. (Basel) 76(1), 34-40 (2001). https://doi. org/10.1007/s000130050539 
16. Wang, B., Luo, C.L., Li, S.H., Chu, Y.M.: Sharp one-parameter geometric and quadratic means bounds for the Sándor-Yang means. Rev. R. Acad. Cienc. Exactas Fís. Nat. Ser. A Mat. RACSAM 114(1), 7-10 (2020). https://doi.org/10.1007/s13398-019-00734-0

17. Wang, J.L., Qian, W.M., He, Z.Y., Chu, Y.M.: On approximating the Toader mean by other bivariate means. J. Funct. Spaces 7, 6082413 (2019). https://doi.org/10.1155/2019/6082413

18. Wang, M.K., Chu, H.H., Chu, Y.M.: Precise bounds for the weighted Hölder mean of the complete p-elliptic integrals. J. Math. Anal. Appl. 480(2), 123388 (2019). https://doi.org/10.1016/j.jmaa.2019. 123388

19. Wang, M.K., He, Z.Y., Chu, Y.M.: Sharp power mean inequalities for the generalized elliptic integral of the first kind. Comput. Methods Funct. Theory 20(1), 111-124 (2020). https://doi.org/10.1007/s40315020-00298-w

20. Witkowski, A.: On Seiffert-like means. J. Math. Inequal. 9(4), 1071-1092 (2015). https://doi.org/10. 7153/jmi-09-83

Publisher's Note Springer Nature remains neutral with regard to jurisdictional claims in published maps and institutional affiliations. 